\title{
Method for the computer-aided design and simulation of hydrogel-based microfluidic chips
}

\author{
Andreas Voigt ${ }^{1 *}$, Jörg Schreiter ${ }^{2}$, Christian Mayr $^{2}$, Andreas Richter ${ }^{1}$ \\ ${ }^{1}$ Chair of Microsystems, Institute of Semiconductors and Microsystems, Faculty of Electrical and Computer Engineering, \\ Technische Universität Dresden, Dresden, Germany, *andreas.voigt@tu-dresden.de \\ ${ }^{2}$ Chair of Highly-Parallel VLSI-Systems and Neuromorphic Circuits, Institute of Circuits and Systems, Faculty of Electrical and \\ Computer Engineering, Technische Universität Dresden, Dresden, Germany
}

\begin{abstract}
Microfluidic chips facilitate the manipulation of nanoliter fluid volumes in order to perform chemical analysis or synthesis and biological cell manipulation. On-chip valves are employed when complex flow control schemes are desired. Stimuli-sensitive hydrogels can be used to create transistor-like microfluidic valves whose opening or closing behavior is determined by the content of chemicals in the liquid. Here, we present a design and simulation method that simplifies the development of hydrogel-based microfluidic chips by reducing the amount of experiments that need to be performed in the lab. Cadence Virtuoso, a tool commonly used for electronic circuit design, is employed as a framework for the implementation of the method. Like in electronics, the microfluidic circuit consists of basic components (channels, valves, pumps) that can be placed and connected with each other in the schematic design interface. The physical-mathematical behavior of these components is implemented in VerilogAMS, a hardware description language. The method was successfully employed in the top-down design of a chemofluidic oscillator. In addition, parts of the design process of the photomasks for chip fabrication were automated by the use of parameterized cells and the SKILL programming language.
\end{abstract}

This article is a summary of the work presented in [1].

\section{Introduction}

Microfluidics is a scientific and engineering discipline that covers the behaviour of fluids (liquids and gases) at the micrometre scale. One endeavour in the field is the construction of labs-on-chips that perform automated chemical, biological or medical laboratory processes on a miniaturized chip. A lab-on-a-chip usually consists of a network of channels and chambers, and inlets and outlets for the liquids and reagents. In addition, the chip can contain microvalves in order to achieve more complicated liquid flow control mechanisms.

Hydrogels are porous polymers that, similar to a sponge, can suck up water and change their size accordingly. Some hydrogels are stimulus-sensitive: their swelling or shrinking depends on the physical or chemical properties of the surrounding liquid, e.g. temperature, $\mathrm{pH}$ value or the presence of specific chemicals. Smart valves ("chemofluidic transistors") can be built by placing a chemosensitive hydrogel in a chamber (figure 1) [2] [3], or by utilizing a membrane that closes an adjacent channel when the hydrogel swells (figure 2) [4]. These valves have the advantage that they do not require any external control and facilitate direct on-chip feedback mechanisms. Employing this internal feedback, chemofluidic transistors have been used to build a microfluidic oscillator [5] and microfluidic logic circuits [6] [7].

Computer-aided design has been a key factor contributing to the development of the advanced state of current microelectronics. Therefore, it seems natural to establish similar methods for microfluidics. Computer-aided microfluidic design, like computer-aided electronic design, can be roughly divided into two categories: Simulation of the physical processes on the chip, and automated drawing of the layout of the masks that are needed for the fabrication of the chip. One approach in simulation is the use of computational fluid dynamic tools that describe the fluid transport in three dimensions and utilize numerical discretisation, e.g. by the finite volume method. Due to the high amount of degrees of freedom, this comes at the cost of a high computational time. Therefore, simplified descriptions have been developed based on the electrohydraulic analogy (pressure corresponds to voltage, flowrate to current) and dimensional reduction [8].

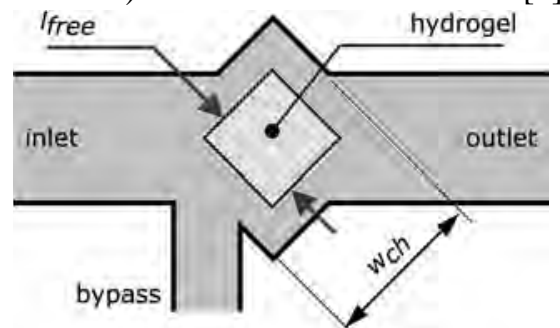

Figure 1: Planar chemofluidic transistor. The hydrogel in the chamber responds to the chemical content in the liquid by swelling or shrinking, leading to opening or blocking of the valve since water can only be transported in the small channels around the gel. 


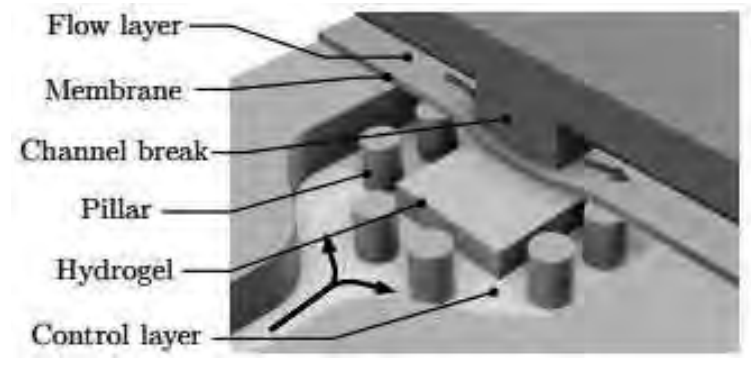

Figure 2: Chemofluidic transistor employing a membrane for the separation of flow layer and control layer. In the swollen state, the gel pushes the membrane against the channel break, blocking the flow in the flow layer. In the shrunken state of the gel, the fluid in the flow layer can pass under the channel break.

Microfluidic chips are then described as circuits of connected basic components, similar to electronic circuits. Another advantage, aside from the significant increase in simulation speed, is the visual representation of the chip functionality and the intuitive usability when designing microfluidic chips on the computer. Modelling languages like Modelica, Simscape or VerilogAMS can be employed for the mathematical description of the microfluidic components. In addition, a design and simulation framework supporting the according language is needed to draw the circuits and compute their behaviour. The Cadence Virtuoso framework with VerilogA was first used by Wang et.al [9] for microfluidic simulations. In this work, we also employ Cadence Virtuoso. However, as the modelling language we use VerilogAMS as it allows combined digital-analogue implementations of component models (AMS = analogue mixed signal) while VerilogA only provides analogue functionality. Compared to Wang et al., the main novelty of our work lies in the timedependent simulation of chemical transport, in the inclusion of chemofluidic transistors, and in the fact that our method was successfully employed in the top-down design of a chemofluidic oscillator [5].

Microfluidic layout automation has been largely driven by the goal to achieve "full automation", the generation of the complete mask layout from an abstract description of the functionality of the chip, e.g. in the Columba 2.0 method by Tseng et al. [10]. However, a survey among microfluidic chip designer conducted by McDaniel et al. [11] indicated that the designers prefer to have more control during all steps of the design process instead of using push-button solutions. Therefore, "semi-automation" of certain steps during layout design seems to be a more adequate approach at the current status of lab-on-a-chip technology. In this line, we have employed the parameterized cells (pCells) and the SKILL language of Cadence
Virtuoso to automate small repetitive and cumbersome steps in the layout process.

\section{Principles and Design Environment}

Like in electronics, to facilitate a schematic circuit representation of a microfluidic chip, a dimensional reduction (1d representation) of the components has to be performed (figure 3). In microchannels, only the effect of the longitudinal direction is taken into account and the pressures are considered constant along the whole cross-section of the channel. Therefore, the channel can be regarded as a component with two terminals. Similarly, all other components are characterized as blocks with terminals, with the inner behaviour of the block described by a VerilogAMS file.

a)

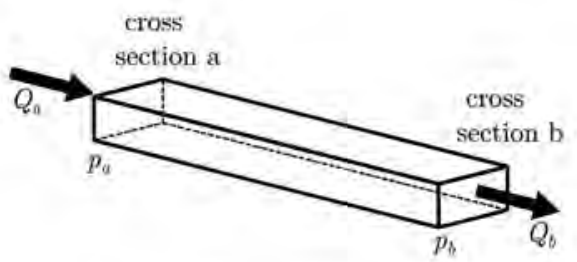

b)

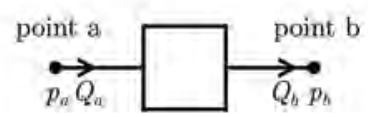

Figure 3: 1d approximation. Microchannels are treated as components with two terminals, each with an applied pressure and a corresponding flowrate.

One main difference between electronics and microfluidics are the chemicals transported in the flow. Therefore, in addition to the pressure-flowrate pair, chemical concentrations need to be implemented in the simulation. We have included chemical concentration as a signal-flow quantity, a feature offered by VerilogAMS and Cadence Virtuoso.

Figure 4 shows a microfluidic schematic. The terminals of components always consist of one input port for the incoming chemical concentration, one output port for the outgoing chemical concentration and one input/output port for the pressure-flowrate pair. Channels are components on their own. In contrast to electronics, the thin "wires" only indicate connectivity and do not correspond to physical entities.

The circuits are entered using the schematic editor of the Cadence Virtuoso design framework. Component models have free parameters that are set when an instance of the component is created. The time-dependent simulation of a circuit is performed by calling the Spectre simulator. 


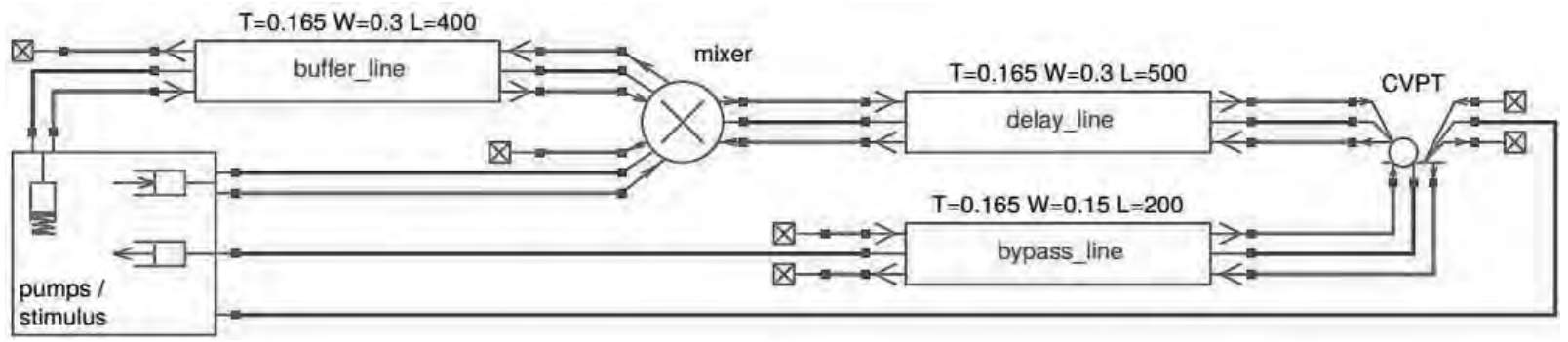

Figure 4: Schematic of the chemofluidic oscillator consisting of pumps, channels (delay line, bypass line and buffer line), a mixer and a chemofluidic transistor (CVPT). The thin lines show the connection of the components and do not correspond to real objects. Blue lines indicate the fluidic quantities (pressure, flowrate); purple lines indicate incoming or outgoing chemical concentrations. Buffer line, delay line and bypass line are all instances of the same component (a microchannel) but with different component parameters.

\section{Components}

Aside from water (which acts as the solvent), the current component models describe the transport of one single chemical species. In the case of the chemofluidic oscillator this species is propan-1-ol, acting as a stimulus for the employed hydrogel.

\subsection{Channels}

Microfluidic channels (figure 5) have two effects on fluid transport. First, they act as resistors with the hydrodynamic resistance $R_{\mathrm{h}}$ depending on the viscosity of the fluid and the channel dimensions (length, height, width). When a pressure difference $p$ is applied at the two terminals of the channel, a flow rate of

$$
Q=\frac{p}{R_{\mathrm{h}}}
$$

results. The second effect is the mechanism by which concentration is transported through the channel. In our model, we neglect diffusion and only account for convection, i.e. the shifting of the position of chemical concentration due to the movement of the fluid.
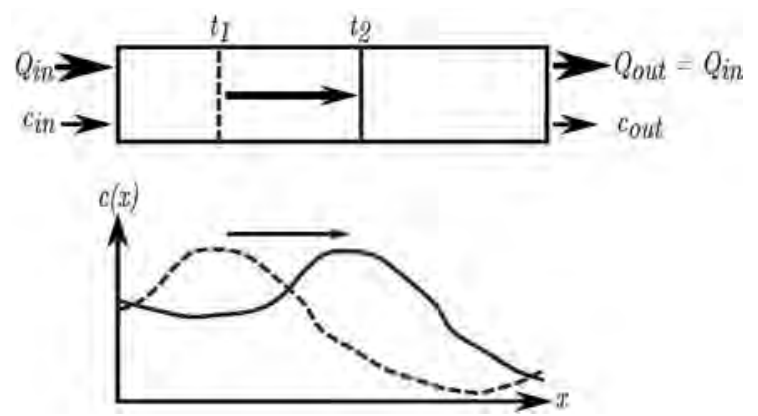

Figure 5: Microfluidic channel. There is an incoming chemical concentration $c_{\text {in }}$ at the inlet and an outgoing concentration $c_{\text {out }}$ at the outlet. The concentration is shifted along the channel in time due to the flow.
In the VerilogAMS model, a combination of analogue and digital modelling is used to account for the two effects. The analogue part describes the resistive behaviour of the channel while the digital part is employed for discretizing the concentration values and for bookkeeping of the internal state of the concentration distribution inside the channel.

\subsection{Mixing Junction}

In a mixing junction (figure 6), two incoming flows of flowrate $Q_{\text {in1 }}$ and $Q_{\text {in2 }}$ carrying concentrations $c_{\text {in } 1}$ and $c_{\text {in2 }}$ are joined. We assume that the outgoing channel is long enough to achieve complete mixing. The outgoing flowrate is simply the sum $Q_{\mathrm{in} 1}+Q_{\mathrm{in} 2}$ of the incoming flowrates and the resulting concentration is calculated by a weighted average:

$$
c_{\mathrm{out}}=\frac{Q_{\mathrm{in} 1} c_{\mathrm{in} 1}+Q_{\mathrm{in} 2} c_{\mathrm{in} 2}}{Q_{\mathrm{in} 1}+Q_{\mathrm{in} 2}} .
$$

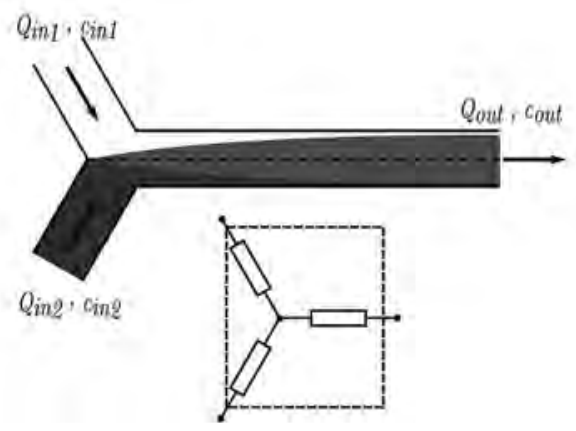

Figure 6: Mixing junction. Two incoming flows of flowrate $Q_{\text {in1 }}$ and $Q_{\mathrm{in} 2}$ carrying concentrations $c_{\mathrm{in} 1}$ and $c_{\mathrm{in} 2}$ are mixed. The outlet delivers the resulting flowrate $Q_{\text {out }}$ and concentration $c_{\text {out }}$.

\subsection{Chemofluidic Transistor}

Here, we focus on the planar chemofluidic transistor (figure 1). The gel in the chamber acts as a hydrodynamic resistor, where the resistance depends on the chamber width $w_{\text {ch }}$ and the current size of the gel. For a free 

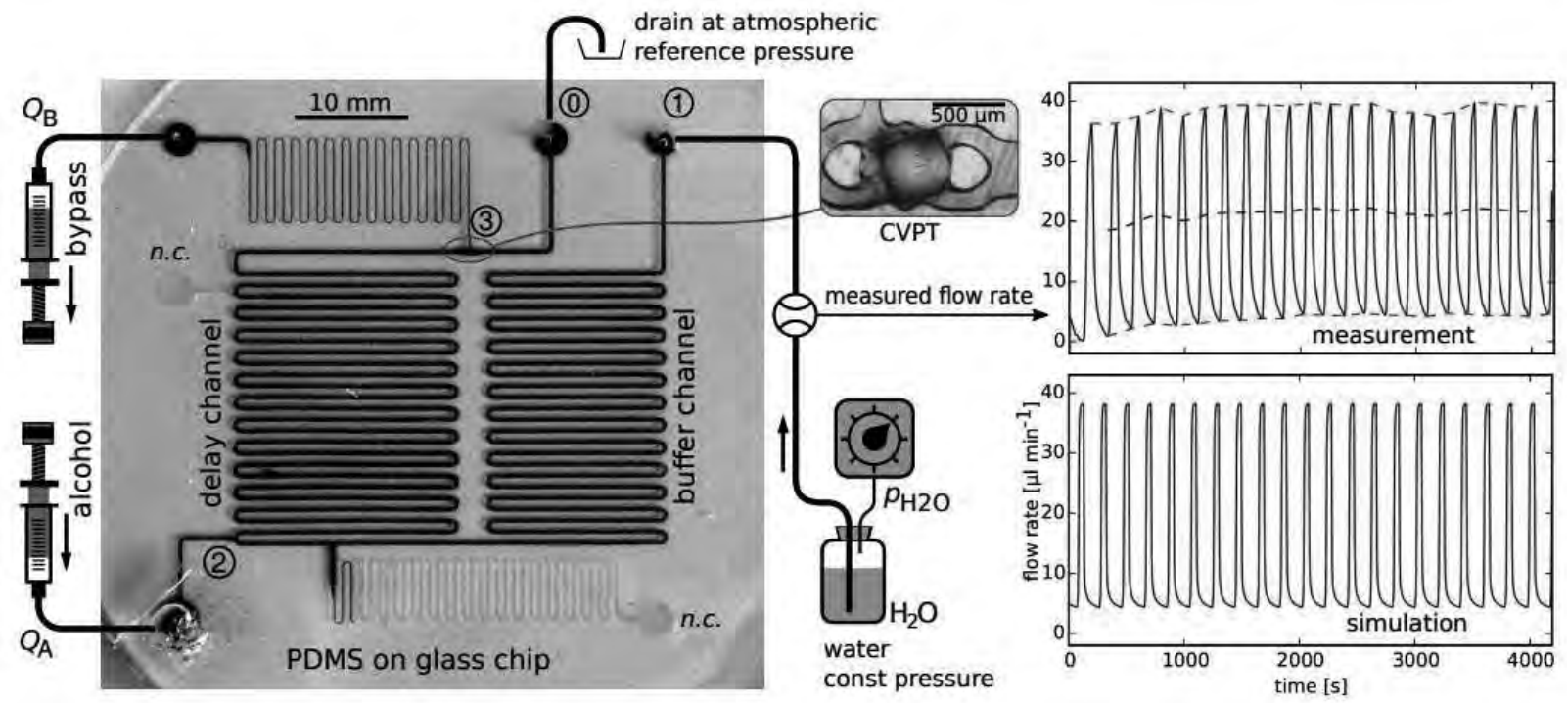

Figure 7: The chemofluidic oscillator is operated by a constant pressure source, by two constant flowrate sources and shows oscillations of flowrates and chemical concentration. This is achieved by the combination of a chemofluidic transistor (CVPT) and a delay channel, providing time-delayed negative feedback. The plots show the measured and the simulated flowrate at the inlet of the buffer channel.

swelling gel (outside of a chamber), the equilibrium size $l_{\text {equ }}(c)$ depends on the concentration of the surrounding liquid (figure 8).

If the current size $l_{\text {free }}$ deviates from this value, the gel will swell or shrink according to a first order differential equation

$$
\frac{\mathrm{d} l_{\text {free }}}{\mathrm{d} t}=\gamma(c) \cdot\left(l_{\mathrm{eq}}(c)-l_{\text {free }}\right) \cdot K\left(l_{\text {free }}\right),
$$

where $\gamma$ is the concentration-dependent swelling rate and $K$ is a size-dependent correction factor that only significantly deviates from 1 for large gel sizes.

For the gel in the chamber we keep book of the current $l_{\text {free }}$ and calculate the hydrodynamic resistance accordingly, where the transistor is blocked for $l_{\text {free }} \geq w_{\mathrm{ch}}$.

The bypass of the chemofluidic transistor is needed to allow the transport of fluid (and hence a chemical signal) to the gel, when the transistor is in its blocked state.

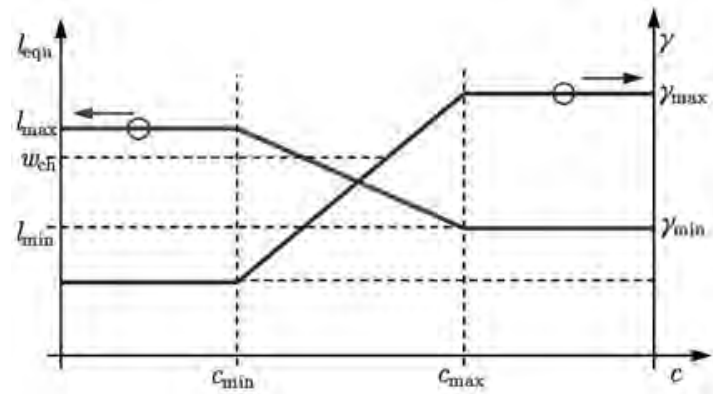

Figure 8: Concentration-dependent equilibrium size $l_{\text {free }}$ and swelling rate $\gamma$ of the hydrogel. The linear piece-wise graphs approximate the experimental behaviour.

\section{Example System: The Chemofluidic}

\section{Oscillator}

The chemofluidic oscillator (figures $4 \& 7$ ) is a microfluidic circuit that is driven by constant fluidic sources and shows periodic oscillations in flowrate and chemical concentration [5]. At the heart of the circuit is a long microfluidic channel (delay channel) leading to a planar chemofluidic transistor (3). The delay channel is fed by a constant flowrate source providing a propan-1-ol solution (2) and a constant pressure source providing pure water (1). Depending on the state of the chemofluidic transistor either the propan-1-ol source or the pure water source dominates. When a solution with high propan-1ol concentration reaches the transistor this will lead to a shrinking of the gel, which in turn will result in a solution with low propan-1-ol concentration being fed into the delay line at (2). After a certain delay time this low concentration will reach the hydrogel, leading to a blocking of the transistor and therefore a solution of high propan-1ol concentration being fed into the delay channel. After a delay time this high concentration will reach the hydrogel, starting a new period of the oscillation.

The chemofluidic oscillator was first conceived conceptually. Then, simulations were used to determine geometric and operational parameters that would lead to a safe oscillation regime. With the determined parameters, the 
mask was drawn for fabrication, and the fabricated microfluidic chip was put into operation. Figure 7 shows the measured and the simulated flowrate at a characteristic position on the chip.

\section{Towards Layout Semi-automation}

As noted by McDaniel et al. [11], microfluidic chip designers prefer to have full control of the design process and, at the current state of the technology, do not trust fully or highly automated layout solutions. Instead, it seems advisable to support them in cumbersome and repetitive day-to-day routines. Along this line, we have developed first steps towards layout semi-automation to alleviate time-consuming processes we encountered during chip design.

The parametric cells (pCells) of Cadence Virtuoso provide the function to define generic layout definitions with a pre-defined shape but with a number of free parameters. In instancing a pCell, the parameters have to be set and the accordingly generated layout block can then be freely placed. This functionality proved especially helpful for the generation of meanders (figure 9) where the channel width, pitch, curvature, the length of the straight lines, the number of meanders, the entrance length and the exit length are set as free parameters.

Additional functionality is provided when augmenting the functionality of pCells by the use of the SKILL programming language. This facilitates the construction of complex, regular (e.g. highly serialized or parallelized) microfluidic structures in lieu of drawing the layout manually [1].

\section{Summary and Outlook}

Our methods allows the design of microfluidic chips employing chemofluidic transistors by placing and routing instances of basic components. The components are modelled by the VerilogAMS hardware description language that provides the flexibility of implementing both analogue and digital behaviour. The simulation shows the time-dependent fluidic quantities (pressure, flowrate), concentration transport and internal states of the components. The method proved useful in the top-down design and parametric dimensioning of a chemofluidic oscillator. In addition, first small steps were taken towards a semi-automation of the layout process by parametric cells.

In the future, we will enhance the component library by including the single-use valves developed in [12]. In addition, it might be worthwhile to investigate whether our method can be transferred to other microfluidic platform technologies, e.g. based on pneumatic valves [13]. For the layout automation, in addition to "little helper" pCells or SKILL scripts, it seems valuable to pursue a closer coupling of the schematic design to the layout process, with the final goal of generating the mask layout directly from the circuit sketch.

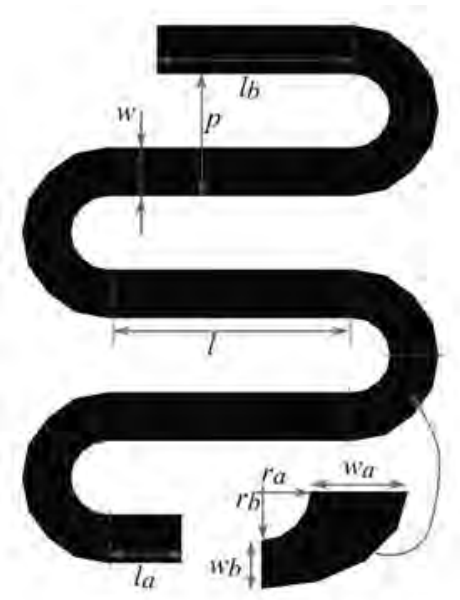

Figure 9: Layout of a parameterized meander channel pCell. The pCell can be instantiated with a chosen set of parameters, providing a flexible and quick meander drawing routine.

\section{References}

[1] A. Voigt, J. Schreiter, P. Frank, C. Pini, C. Mayr, A. Richter, Method for the Computer-aided Schematic Design and Simulation of Hydrogel-based Microfluidic Systems, IEEE Transactions on Computer-Aided Design of Integrated Circuits and Systems, vol. 39, no. 8, pp. 1635-1648, Aug. 2020. DOI: 10.1109/TCAD.2019.2925354

[2] D. J. Beebe, J. S. Moore, J. M. Bauer, Q. Yu, R. H. Liu, C. Devadoss, B.-H. Jo, Functional hydrogel structures for autonomous flow control inside microfluidic channels, Nature, vol. 404, no. 6778, pp. 588-590, Apr. 2000.

[3] K.-F. Arndt, D. Kuckling, A. Richter, Application of sensitive hydrogels in flow control, Polymers for Advanced Technologies, vol. 11, nos. 8-12, pp. 496-505, Aug. 2000.

[4] P. Frank, D. Gräfe, C. Probst, S. Haefner, M. Elstner, D. Appelhans, D. Kohlheyer, B. Voit, and A. Richter, Autonomous Integrated Microfluidic Circuits for Chip-Level Flow Control Utilizing Chemofluidic Transistors, Advanced Functional Materials, vol. 27, no. 30, p. 1700430, 
Aug. 2017.

[5] G. Paschew, J. Schreiter, A. Voigt, C. Pini, J. P. Chávez, M. Allerdißen, U. Marschner, S. Siegmund, R. Schüffny, F. Jülicher, and A. Richter, Autonomous Chemical Oscillator Circuit Based on Bidirectional Chemical-Microfluidic Coupling, Advanced Materials Technologies, vol. 1, no. 1, p. 1600005, Apr. 2016.

[6] A. Voigt, R. Greiner, M. Allerdißen, A. Richter, S. Henker, M. Völp, Towards computation with microchemomechanical systems, International Journal of Foundations of Computer Science, vol. 25, no. 4, pp. 507-523, Jun. 2014.

[7] P. Frank, D. Gräfe, C. Probst, S. Haefner, M. Elstner, D. Appelhans, D. Kohlheyer, B. Voit, A. Richter, Autonomous integrated microfluidic circuits for chip-level flow control utilizing chemofluidic transistors, Advanced Functunal Materials, vol. 27, no. 30, p. 1700430., Aug. 2017

[8] R. Zengerle, M. Richter, Simulation of microfluid systems, Journal of Micromechanics and Microengineering, vol. 4, no. 4, pp. 192-204, Dec. 1994.

[9] Y. Wang, Q. Lin, T. Mukherjee, Composable Behavioral Models and Schematic-Based Simulation of Electrokinetic Lab-on-a-Chip Systems, IEEE Transactions on Computer-Aided Design of Integrated Circuits and Systems, vol. 25, no. 2, pp. 258-273, Feb. 2006.

[10] T. Tseng, M. Li, D. N. Freitas, T. McAuley, B. Li, T. Ho, I. E. Araci, and U. Schlichtmann, Columba 2.0: A CoLayout Synthesis Tool for Continuous-Flow Microfluidic Biochips, IEEE Transactions on Computer-Aided Design of Integrated Circuits and Systems, vol. 37, no. 8, pp. 1588-1601, Aug. 2018.

[11] J. McDaniel, W. H. Grover, and P. Brisk, The case for semi-automated design of microfluidic very large scale integration ( $m V L S I$ ) chips, in Design, Automation \& Test in Europe Conference Exhibition (DATE), 2017, Mar. 2017, pp. 1793-1798.

[12] R. Greiner, M. Allerdissen, A. Voigt, A. Richter, Fluidic microchemomechanical integrated circuits processing chemical information, Lab on a Chip, vol. 12, no. 23, 5034-5044, 2012

[13] T. Thorsen, S. J. Maerkl, S. R. Quake, Microfluidic large-scale integration, Science, vol. 298, no. 5593, pp. 580-584, Oct. 2002. 